LaRk, K. G., MaAloe, O. \& Rostock, O. (1955). J. gen. Microbiol. 13, 318-326

\title{
Cytological Studies of Nuclear Division in Salmonella typhimurium
}

\author{
BY K. G. LARK, O. MAALØE AND O. ROSTOCK
}

Statens Seruminstitut, Copenhagen, Denmark

\begin{abstract}
SUMMARY: Physiological studies recently led to the development of a technique for obtaining synchronous nuclear division in Salmonella typhimurium. By using this method we have attempted to identify the morphological changes characteristic of nuclear division. The picture obtained is that of a gradual shift from a round nuclear body via 'wedge'- and 'bar'- to 'dumbbell'-like configurations. Mitotic patterns were not observed in these small cells. An indication was obtained that at an early stage of nuclear division the nucleus has already doubled with respect to at least one of its physiological functions. It is discussed to what extent the present studies by selective staining may be used to identify the state of the nucleus as observed by electron microscopy.
\end{abstract}

In recent years many cytological observations of the bacterial nucleus have been reported, leading to the putting forward of different theories about the cytology of nuclear division in bacteria (Robinow, 1946; DeLamater, 1951). Until now it has been impossible to test such theories rigorously because cultures in which nuclear division occurs in a random fashion do not permit the identification of the physiological states of the nuclei under observation. With a view to removing this limitation, a system was developed in this laboratory for synchronizing nuclear division in large populations of Salmonella typhimurium (Maaløe \& Lark, 1954; Lark \& Maaløe, 1954). By studying populations of synchronized cells, fixed at various times relative to the beginning and end of nuclear division, it was thought that it might thus be possible to identify a cytological sequence of events for the division of the bacterial nucleus. The present paper presents a cytological study of this system by means of selective staining techniques, as a preliminary to a more detailed study, by means of electron microscopy of serial sections, which is now being carried out by Dr A. Birch-Andersen. Preliminary results of this latter investigation are contained in the next paper (Birch-Andersen, 1955).

\section{METHODS}

The properties of the strain of Salmonella typhimurium, the liquid and solid media used, and the induction of synchronous nuclear division by means of the single temperature shift method were described previously (Lark \& Maaløe, 1954). A broth culture of $S$. typhimurium which had been growing exponentially at $25^{\circ}$ for more than ten generations was allowed to reach a density of $10^{7}$ cells $/ \mathrm{ml}$. At this time aeration was started to maintain optimal aerobic growth conditions. When the density had increased to about $5 \times 10^{7}$ cells $/ \mathrm{ml}$. the temperature of the culture was raised to $37^{\circ}$ by the addition of an 
appropriate volume of broth warmed to $90^{\circ}$. The culture was then grown at $37^{\circ}$ with aeration and diluted at intervals to maintain a titre of approximately $5 \times 10^{7}$ organisms $/ \mathrm{ml}$. Samples were removed at intervals for colony counts and for cytological examination. The colony count growth curve thus obtained was identical with that found previously (Maaløe \& Lark, 1954). This finding, in addition to the cytological observations to be presented, demonstrated that synchronous nuclear and subsequent synchronous cellular division had indeed been induced.

Samples $(4 \mathrm{ml}$.) were removed for cytological observation and immediately mixed with $1 \mathrm{ml}$. of a $5 \% \mathrm{OsO}_{4}$ solution to obtain rapid fixation. After standing for $3 \mathrm{~min}$. at room temperature this solution (now $1 \%$ in $\mathrm{OsO}_{4}$ ) was diluted to $40 \mathrm{ml}$. with broth to avoid overfixation, and centrifuged. Following centrifugation the cells were resuspended in $\mathbf{0 . 2} \mathbf{~ m l}$. broth, and samples of this suspension were immediately spotted on the surface of a dry agar plate. After the samples had dried into the agar surface, the Petri dish was inverted over distilled water and thus kept until impression smears could be made on coverslips. The preparations were hydrolysed for $1 \mathrm{~min}$. in $\mathrm{N}-\mathrm{HCl}$ at $60^{\circ}$ (the previously ascertained optimum time of hydrolysis for nuclear staining of this organism), rinsed in distilled water and stained for $2 \mathrm{hr}$. at room temperature in $\mathbf{0 . 2 5} \%(\mathrm{w} / \mathrm{v})$ thionine containing $5 \mathrm{mg} . / \mathrm{ml}$. thionyl chloride (DeLamater, 1952). After this the preparations were again rinsed in distilled water, dehydrated in absolute ethanol at $-\mathbf{7 0}$ for $\mathbf{2 4} \mathrm{hr}$., and passed through ethanol (twice) and xylene (three times) at room temperature before being mounted in Canada Balsam.

Control stainings of non-synchronized cultures showed that the liquidfixation technique, employed to ensure rapid fixation, gave results identical with those obtained by fixation over $1 \%$ osmium tetroxide vapour for $3 \mathrm{~min}$. It should be noted, however, that cells fixed in liquid medium clumped during centrifugation, making it difficult to find fields which were rich in individual cells (cf. Pl. 1, fig. $b$ ).

Because of the small size of the organisms studied $(0 \cdot 5-0 \cdot 7 \mu$. wide, $1-4 \mu$. long) a Reichert microscope equipped with an 'Anoptral' condenser, a 'Fluorit' objective $(\times 100)$ and a Plan-ocular $(\times 12)$ was used. The wide ringaperture of the condenser (normally used for phase-contrast microscopy with oil-immersion) was adjusted so that light which penetrated the specimen obliquely reached the periphery of the objective leaving the centre region unused. This arrangement, which was suggested by A. Findeis, of the Reichert Company, gives excellent resolution and contrast.

\section{RESULTS}

Before and after the shift in temperature used to induce nuclear division, samples were removed from the culture, fixed and stained. In repeated experiments the most striking change noticed when first looking at the various preparations was the large increase in the proportion of quadri-nucleate cells present in the population. This increase began at $c .10 \mathrm{~min}$. after the tempera- 
ture shift, a maximum frequency of quadri-nucleate cells being attained by c. $20 \mathrm{~min}$. after the temperature shift. After $20 \mathrm{~min}$. the frequency of quadrinucleate cells gradually returned to the low value observed before raising the temperature. The return to this state was completed at 50-60 min. after the temperature shift. Pictures of cells fixed before the temperature shift (consisting predominantly of bi-nucleate cells), and of cells from the synchronized population fixed 20 min. after the temperature shift (showing a large proportion of quadri-nucleate cells) are given in Pl. 1, figs. $a$ and $b$.

Between the extremes just described more subtle changes in the nuclear bodies were observed. In exponentially growing cultures of Salmonella typhimurium at $25^{\circ}$ (generation time $=50 \mathrm{~min}$.) or at $37^{\circ}$ (generation time $=$ $22 \mathrm{~min}$.) the nuclei appeared predominantly as compact dots, whereas, during the period between the temperature shift and the increase in number of quadri-nucleate cells, a large proportion of the cells showed other nuclear configurations. These configurations could usually be assigned to one of three arbitrary classes designated as wedge, bar and dumbbell structures; the four types of nuclear configuration are illustrated diagrammatically in Fig. 1, and examples of nuclei of the different types are presented on Pl. 2.

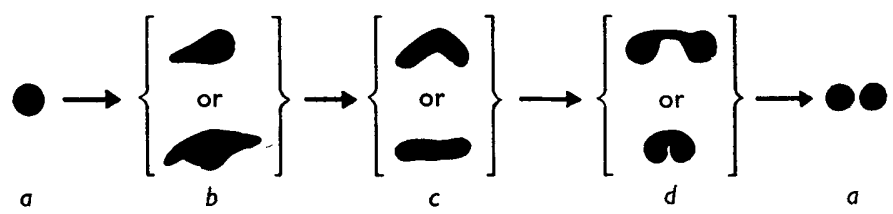

Fig. 1. Schematic representation of the four configurations- $\operatorname{dot}(a)$, wedge $(b)$, bar $(c)$ and dumbbell $(d)$-successively assumed by the dividing nucleus of Salmonella typhimurium. The two different shapes representing each of the classes $b, c$ and $d$ roughly illustrate the variations observed.

The predominance of dot structures in randomly dividing cultures, as compared to the low frequency found during the period of induced nuclear division, at once suggested that in Salmonella typhimurium this configuration represented nuclei not involved in division. Moreover, the preliminary survey of preparations fixed after the temperature shift indicated that the frequencies of bi-nucleate cells containing structures other than dots rose and fell in a systematic way which suggested that nuclear division proceeded from dots via wedge and bar to dumbbell structures. This sequence is illustrated in Fig. 1. With this general impression in mind, an enumeration of cells containing the four different nuclear structures was carried out, using the micrographs obtained with the high-resolution system described above. For practical reasons the cells were classified as follows: $(a)$ cells containing 'dots' only; (b) cells containing at least one 'wedge' but no 'bar' or 'dumbbells'; $(c)$ cells containing at least one bar but no dumbbells; $(d)$ cells with one or more dumbbells. The results obtained with this classification are given in Table 1; typical fields showing high frequencies of cells with changed nuclei are presented on Pl. 1, figs. $c$ and $d$, which should be compared with Pl. 1, fig. $a$.

For the interpretation of the data in Table 1 it is important to notice that the classification method is biased in favour of cells which contain bars and 
dumbbells. Thus an increase in the frequency of bar-cells will lower the frequency of wedge-cells for each cell containing a wedge and a bar, while a rise in the frequency of dumbbell cells may depress the frequencies of both wedgeand bar-cells. Conversely, increases in the frequencies of wedge- or bar-cells will be picked up and correctly registered as long as the cells do not at the same time contain bars or dumbbells respectively. These considerations show that the above simple classification may be used to test the hypothesis that during division the nuclear configuration changes from dot via wedge and bar to dumbbell.

Table 1. Classification of cells according to number of nuclei and to nuclear configuration

\begin{tabular}{|c|c|c|c|c|c|c|c|c|c|}
\hline \multirow[b]{2}{*}{$\begin{array}{c}\text { Time of } \\
\text { sampling } t\end{array}$} & \multicolumn{4}{|c|}{ Bi-nucleate cells } & \multicolumn{4}{|c|}{ Quadri-nucleate cells } & \multirow[b]{2}{*}{$\begin{array}{c}\text { Total of } \\
\text { cells scored }\end{array}$} \\
\hline & $\begin{array}{c}a \\
\%\end{array}$ & $\begin{array}{r}b \\
\%\end{array}$ & $\begin{array}{c}c \\
\%\end{array}$ & $\begin{array}{r}d \\
\%\end{array}$ & $\begin{array}{c}a \\
\%\end{array}$ & $\begin{array}{c}b \\
\%\end{array}$ & $\begin{array}{c}c \\
\%\end{array}$ & $\begin{array}{l}d \\
\%\end{array}$ & \\
\hline-1 & 74 & 8 & 6 & 3 & 8 & 1 & 0 & ) & 336 \\
\hline 1 & 66 & 8 & 7 & 6 & 13 & 0 & 0 & 0 & 246 \\
\hline 3 & 48 & 20 & 9 & $\mathbf{9}$ & 12 & 2 & 0 & 0 & 252 \\
\hline 4 & 41 & 32 & 8 & 8 & 11 & $\mathbf{1}$ & 0 & 0 & 226 \\
\hline 5 & 40 & 25 & 16 & 5 & 14 & 0 & 0 & 0) & 183 \\
\hline 6 & 22 & 33 & 21 & 15 & 6 & $\mathbf{1}$ & 1 & 1 & 100 \\
\hline 7 & 15 & 45 & 21 & 9 & 10 & 0 & 0 & ) & 50 \\
\hline 8 & 27 & 18 & 17 & 28 & 8 & 1 & 0 & 1 & 272 \\
\hline 9 & 26 & 16 & 14 & 27 & 16 & $\mathbf{1}$ & 0 & 0) & 327 \\
\hline 11 & 30 & 15 & 8 & 35 & 12 & 0 & 0 & () & 74 \\
\hline 13 & 30 & 13 & 12 & 19 & 26 & 0 & 0 & 0 & 146 \\
\hline 16 & 31 & 7 & 7 & 22 & 27 & 3 & 3 & 0 & 291 \\
\hline 20 & 23 & 8 & 8 & 22 & 26 & 10 & 2 & 1 & 308 \\
\hline 25 & 12 & 8 & $\tilde{5}$ & 25 & 20 & 22 & 3 & 5 & 190 \\
\hline 30 & 16 & 9 & 6 & 26 & 25 & 12 & 4 & 2 & 302 \\
\hline 35 & 27 & 5 & 4 & 33 & 9 & 9 & 5 & 8 & 100 \\
\hline 60 & 56 & 14 & 8 & 13 & 9 & 0 & 0 & o & 209 \\
\hline
\end{tabular}

The cell-classes $a, b, c$ and $d$ are those described on p. 320; viz. cells containing dot, wedge, bar and dumbbell structures respectively. Cells containing two dots and one dumbbell were arbitrarily classified as bi-nucleate class $d$ cells. When comparing the data presented here with previous statements about the high frequencies of quadri-nucleate cells attained around 16-25 min. (Lark \& Maaløe, 1954), it should be noted that, previously, bi-nucleate dumbbell cells were scored as quadri-nucleate cells.

Another problem of importance for the interpretation of our data should be mentioned. In order to differentiate between the four types of nuclear configurations it is necessary to work at the limit of optical resolution, which results in considerable differences between the results obtained when different observers try to classify cells according to our scheme. Thus the data presented in Table 1, which were obtained by one of the authors (K.G. L.), differ markedly from similar data obtained by another author (O.M.). However, the two sets of figures differ systematically in such a way that the numbers of wedge, bar and dumbbell structures scored by one observer were consistently higher than those scored by the other. The times when the observed frequencies changed, as well as the magnitudes of these changes, thus seem to be relatively independent of the observer, whereas the absolute values for the frequencies are not. 
To bring out clearly the important changes in the frequencies of cells containing dot, wedge, bar and dumbbell structures respectively (the cell-classes $a-d)$, data from Table 1 were used to construct Fig. 2 in which the frequencies for bi-nucleate cells for the samples taken until $20 \mathrm{~min}$. after the temperature shift are plotted as functions of time. In addition, the frequencies of quadrinucleate cells have been plotted. As may be seen the frequency of wedge structures $(b)$ began to rise 2 min. after the temperature shift and continued to do so for the next 4 min. Similar increases in bar and dumbbell structures $(c$ and $d)$ were initiated 2 and $4 \mathrm{~min}$., respectively, after the rise in wedges $(b)$. During the increase in dumbbell structures $(d)$, the frequencies of wedges $(b)$

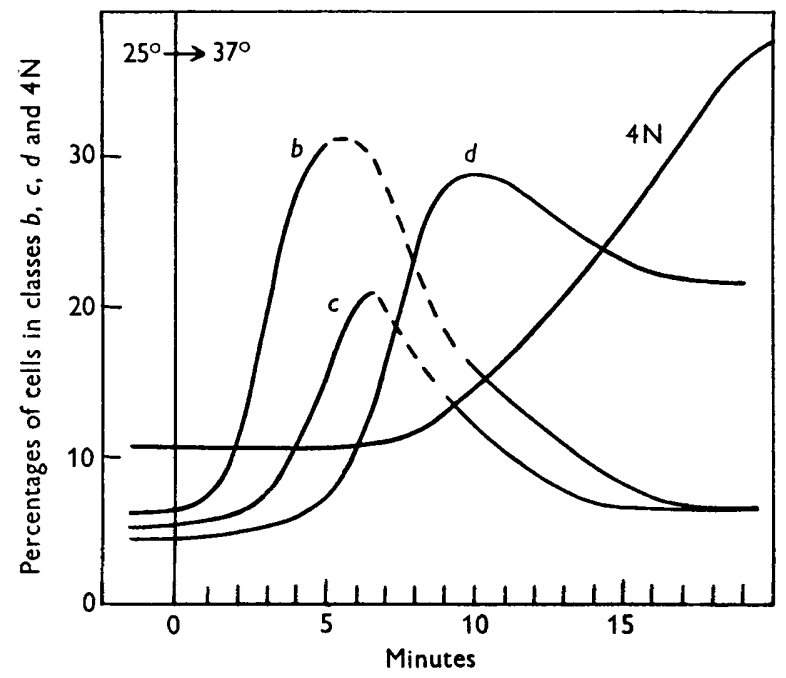

Fig. 2. The changing frequencies of nuclear configurations during the period after the temperature shift. The classes $b, c$ and $d$ are those schematically presented in Fig. 1. Class ' $4 N$ ' is the class of cells with four distinct nuclei. The stippled curve sections indicate that in these regions the shape of curves $b$ and $c$ are influenced by the simultaneous rise in curves $c$ and $d$. (See text on p. 322.)

and bars $(c)$ decreased. The steepness of these decreases is undoubtedly due to some extent to the method of classification used; however, the frequency of bi-nucleate cells which contained wedges $(b)$ continued to decline after the frequency of dumbbells $(d)$ had ceased to increase. This would seem to indicate a real decrease in the frequency of bi-nucleate cells which contain wedge structures.

Finally, at 9-10 min. after the temperature shift, i.e. 2-4 min. following the time at which the frequency of dumbbells $(d)$ began to increase, the frequency of cells containing four distinct nuclei began to increase and reached a constant high level within the next $10 \mathrm{~min}$.

From the progression of times at which the frequencies of wedge, bar and dumbbell structures began to increase, and from the time at which the frequency of quadri-nucleate cells began to increase, it may be concluded that the cytological sequence of events during nuclear division in Salmonella typhi- 
murium is that proposed in Fig. 1. Thus the dot structure, or compact nuclear mass grows and extends thin branches in one or two directions (wedge); the nuclear mass then distributes itself more uniformly (bar), finally coalescing into two terminal globules (dumbbell) which break up to give two compact nuclear masses.

In the period from 16 to $25 \mathrm{~min}$. after the temperature change a slow increase in the frequency of quadri-nucleate cells with wedge structures takes place. An apparent increase in bar and dumbbell structures in quadri-nucleate cells was also noted; a continued high frequency of bi-nucleate dumbbell structures was also seen during this period while, as was mentioned earlier, the frequency of bi-nucleate cells with wedge structures decreased during this period. The only obvious source of bi-nucleate cells containing dumbbells is the quadrinucleate cells containing wedge structures. This would imply that nuclear division of the quadri-nucleate cells proceeded up to the 'wedge' stage in quadri-nucleate form, at which time the cells divided, and nuclear division was completed in the bi-nucleate stages, thus preserving for some time a high proportion of quadri-nucleate cells. This conclusion is born out by the observation that many quadri-nucleate cells whose nuclei were either in the 'dot' or 'wedge' stage showed transverse dark structures which divided the cell into two bi-nucleate half-cells. These structures, which looked like the septa observed in other cells (e.g. by Murray \& Robinow, 1952), were at the limit of the resolving power of the optical system used (see Pl. 1, fig. $d$ ).

Finally some mention of cell size should be made. In the random cultures at 25 and $37^{\circ}$, the bi-nucleate dot-cells $(a)$ were $1-1.5 \mu$. long and $0 \cdot 5-0 \cdot 7 \mu$. wide. Following the induction of nuclear division the width of the cells remained fairly constant, but there was steady increase in length of the binucleate cells, the average cell length being $1 \cdot 2,1 \cdot 4,1 \cdot 6$ and $2 \cdot 1 \mu$. at $3,7,11$ and 20 min., respectively. From this it seems that the wedge structure predominantly existed in cells of length $1 \cdot 4 \mu$. or more, while dumbbells were formed chiefly in cells of length $1 \cdot 6 \mu$. or more. The length of cells which contained wedge or dumbbell structures, taken from random cultures, agreed with this idea.

\section{DISCUSSION}

The foregoing studies were undertaken in an effort to elucidate the sequence of visible changes during nuclear division. It was felt that by studying a bacterial population in which synchronous nuclear division had been induced, it would be possible to recognize definite cytological stages in the division process. This attempt has met with only a limited success. A crude series of successive cytological stages has been ascertained for nuclear division in Salmonella typhimurium. These stages are represented in Fig. 1 as 'dot', 'wedge', 'bar' and 'dumbbell' structures, and this classification could not be refined because of the limited resolution of the optical system used. Indeed, as has been pointed out, many borderline cases have been observed, making unequivocal classification of the nuclear configurations impossible. As may 
be seen from Pl. 2, the 'bar' class contains transition stages between 'wedge' and 'dumbbell' configurations.

From the data to hand, it seems that the most reasonable description of the sequence in nuclear division in Salmonella typhimurium is as follows: nuclear division can be pictured as the gradual transition from one compact body via the more irregular structures described as 'wedge', 'bar' and 'dumbbell' into two distinct round bodies; this picture would tend to agree with that advanced by Robinow (1946). Nuclear configurations of the types claimed by DeLamater (1951) to represent mitotic patterns were observed occasionally. However, no accumulation of such configurations was found during the transition from the bi- to the quadri-nucleate state.

The elucidation of the detailed mechanism of the transition from the two- to the four-dot stage must await studies by means of electron microscopy. This end would seem to be much advanced by the present studies. As has been shown (Birch-Andersen, 1955) serial sections of Salmonella typhimurium may be superimposed on one another to permit a high resolution composite view of the cell. From the overall appearance of such a composite picture, it should be possible to establish whether the nucleus in question is of a dot, wedge, bar or dumbbell configuration and thus roughly establish its position in the sequence of nuclear division. Based on this procedure for identification of the nuclear state an analysis of the individual sections may permit a detailed mapping of the nuclear structures.

Because of the lack of precision in differentiating between the dot, wedge, bar and dumbbell configurations it is difficult to correlate the cytological changes observed after the induction of nuclear division with physiological changes, such as the ability to become lysogenized upon infection with a temperate phage (Maaløe \& Lark, 1954). Nevertheless, certain qualitative comparisons may be made. In Fig. 3 the characteristic change in the lysogenization frequency is illustrated by the fully drawn curve (1) which is to be compared with the dotted curves ( 2 and 3 ) which show the changes in the cumulative frequencies of bi-nucleate cells containing nuclei representing early and late stages of division respectively; viz. curve 2 which shows the cumulative frequencies of cells containing at least one nucleus which is not of the dot type, and curve 3 which shows the frequencies of cells containing at least one dumbbell. As Fig. 3 shows, curve 2 rises first and this rise is soon followed by the increase in lysogenization frequency, whereas the rise in curve 3 sets in 2-3 min. later. We previously advanced the hypothesis that the doubling of the number of nuclei/cell is reflected, physiologically, in a doubling of the lysogenization frequency (Maaløe \& Lark, 1954). In the light of the cytological evidence now at hand we may conclude that soon after a nucleus has assumed the wedge configuration it reaches a state in which neither the process of lysogenization nor lowering of the temperature to $25^{\circ}$ (at which temperature phage infection takes place) interfere with nuclear division.

Finally we would like to discuss the septate appearance of quadri-nucleate cells during the period in which the colony count rises abruptly. As has been mentioned, such 'septa' appear on the limit of resolution of the optical system 
and the difficulties encountered in seeing them might be due to a failure to employ cell-wall stains. However, it is of interest to note that no structure of a septum-like nature has been observed in electron micrographs of sectioned cells taken from this period of a synchronized typhimurium system, although many cells have been observed in various stages of cellular division. (Ranging from a slight pinching to an almost complete severance of the cell, by constriction, into two cells; Birch-Andersen, Lark, Maaløe, Sjöstrand, 1954.)

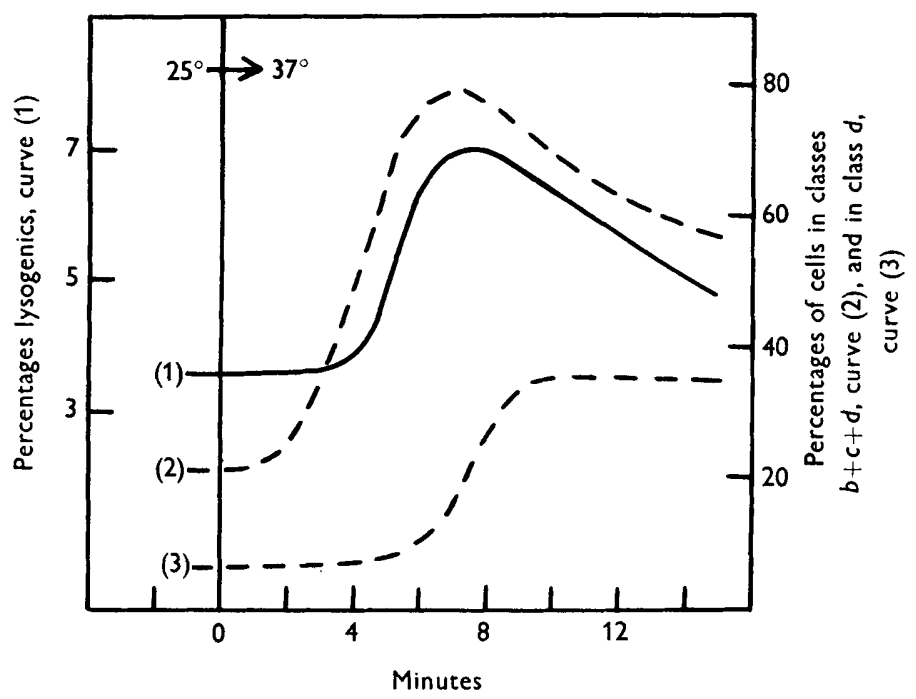

Fig. 3. The frequency of lysogenization compared with the frequencies of cells with actively dividing nuclei. Curve 1, illustrating the abrupt rise in the frequency of lysogenization, is transcribed from a previous paper (Maaløe \& Lark, 1954). Curve 2 illustrates the rise in the cumulated frequency of bi-nucleate cells containing wedge, bar or dumbbell structures, i.e. cells with actively dividing nuclei. Curve 3 represents the rise in the frequency of bi-nucleate cells with one or two dumbbells.

From this, it seems that the septate appearance of the cell is not due to a septum being formed across the cell before division but is due to some optical artefact, which arises because of the constricted character of the cell. True septa have been demonstrated conclusively by means of electron microscopy of thin sections (Chapman \& Hillier, 1953) in Bacillus cereus which, however, possesses a thick and clearly differentiated cell wall which is altogether lacking in Salmonella typhimurium. In view of this, it may be wise to re-examine by means of thin section electron microscopy many of the septum-type structures reported in the literature.

We are grateful to Dr N. O. Christensen for the loan of his microscopical equipment.

Our best thanks are due to Doctors M. H. Adams, W. L. Barksdale and B. A. D. Stocker, who read the manuscript and suggested important improvements. One of us (K.G.L) is a Fellow of the American Cancer Society, upon recommendation of the Committee on Growth, National Research Council. 


\section{REFERENCES}

Birch-Andersen, A. (1955). Reconstruction of the nuclear sites of Salmonella typhimurium from electron micrographs of serial sections. J. gen. Microbiol. 13, 327.

Birch-Andersen, A., Lark, K. G., Maaløe, O. \& Sü̈strand, F. S. (1954). A study of sections of bacteria undergoing synchronous nuclear division. Proc. Int. Conf. Electron Microscopy. London, paper no. 72.

Chapman, G. B. \& Hillier, J. (1953). Electron microscopy of ultra-thin sections of bacteria. I. Cellular division in Bacillus cereus. J. Bact. 66, 362.

Delamater, E. D. (1951). Evidence for the occurrence of mitosis in bacteria. Science, 113, 477.

Delamater, E. D. (1952). A consideration of the new methods for the demonstration of nuclear structure in bacteria and other micro-organisms. Mikroskopie, 7 , 358.

LARK, K. G. \& MAaloE, O. (1954). The induction of cellular and nuclear division in Salmonella typhimurium by means of temperature shifts. Biochim. Biophys. Acta, 15, 345.

MAALøE, O. \& LARK, K. G. (1954). A study of bacterial populations in which nuclear and cellular divisions are induced by means of temperature shifts. In Recent developments in Cell Physiology. Symp. Colston Res. Soc. 7, 159.

Murray, R. G. E. \& Robinow, C. F. (1952). A demonstration of the disposition of the cell wall of Bacillus cereus. J. Bact. 63, 298.

Robinow, C. F. (1946). Nuclear apparatus and cell structure of rod-shaped bacteria. Addendum to Dubos: The Bacterial Cell. Harvard University Press.

\section{EXPLANATION OF PLATES}

Plate 1: Salmonella typhimurium.

Cells fixed and stained selectively for DNA at different times relative to the temperature shift. Magnification, $\times \mathbf{3 4 0 0}$.

Fig. $a$. Predominantly bi-nucleate cells, as seen in a culture growing exponentially at $25^{\circ}$. Cells with 'dots' only, class $a$.

Fig. $b$. Predominantly quadri-nucleate cells, as seen $20 \mathrm{~min}$. after raising the temperature to $37^{\circ}$. Note the unfortunate tendency to clumping after centrifugation of the fixed cells. Cells with one or more 'wedges', class $b$.

Figs. $c, d$. Cells fixed 11 and $30 \mathrm{~min}$. after the temperature shift, respectively. Fields showing many cells with actively dividing nuclei, i.e. nuclei of the classes $b, c$ or $d$, illustrated on Pl. 2. Cells marked with $\nearrow$ contain 'bars', class $c$. Cells with one or more 'dumbbells', class $d$.

\section{Plate 2}

Examples of cells of classes $a, b, c$ and $d$; see p. 320-321. Magnification, $\times 5100$. 
Journal of General Microbiology, Vol. 13, No. 2
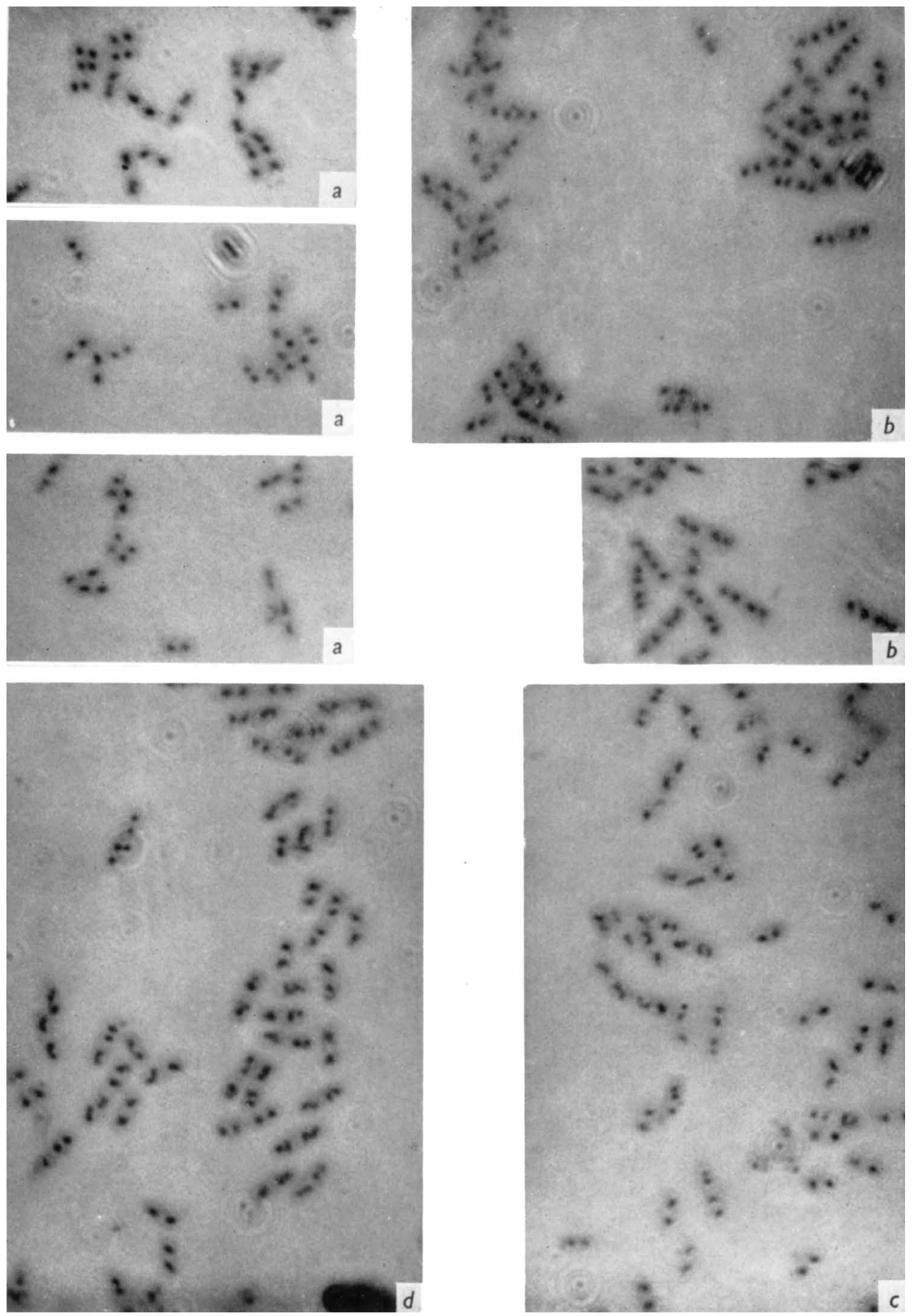

K. G. Lark, O. MaAløe \& O. Rostock-Nuclfar division in S. ty PhinuriUm. Plate 1

(Facing $p .326$ ) 
Journal of General Microbiology, Vol. 13, No. 2
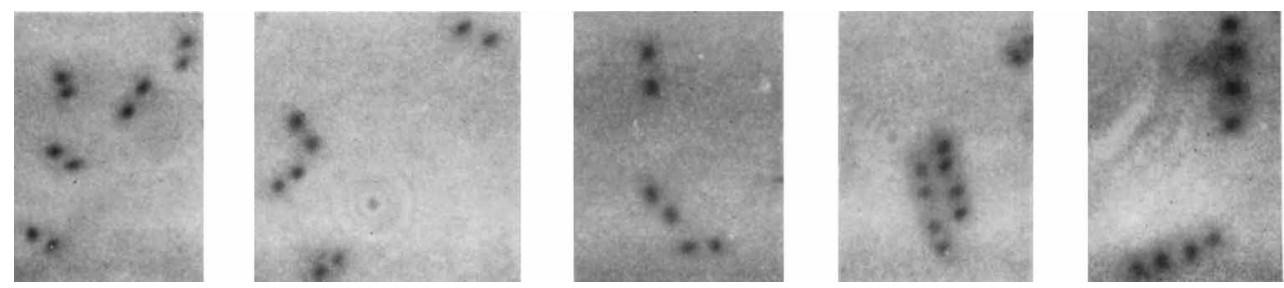

(a) Cells with dots only, class a.
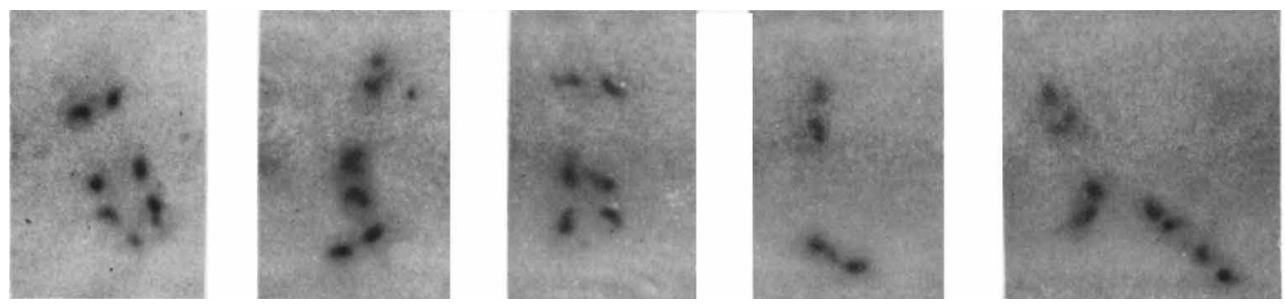

(b) Cells with one or more wedges, clas; b.
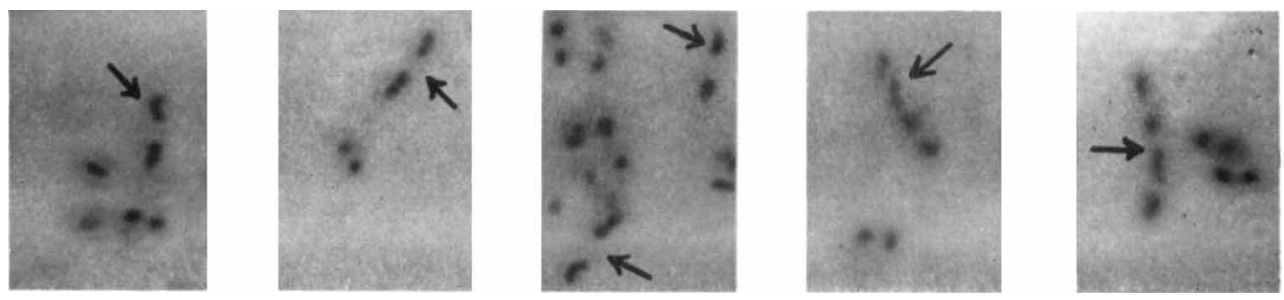

(c) Cells marked with $\nearrow$ contain bars, class $c$.
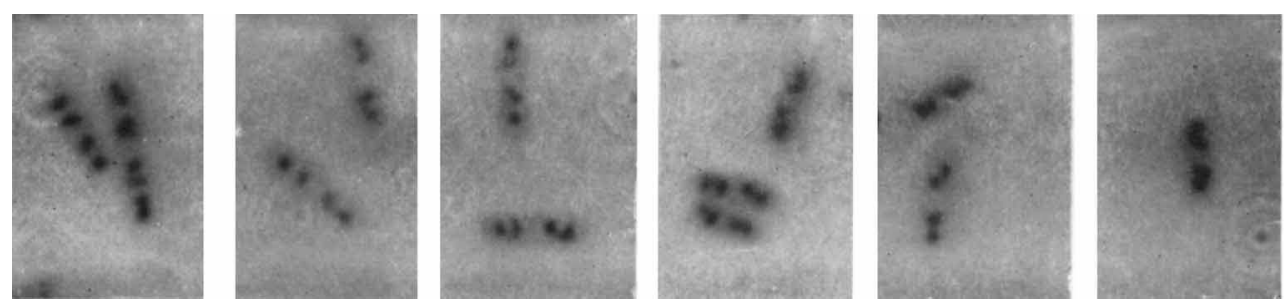

(d) Cells with one or more dumbbells, class d.

K. G. Lark, O. Maaløe \& O. Rostock-Nuclear division in S. typhinurlum. Plate 2 